doi:10.13108/2021-13-3-95

\title{
CONTINUOUS-DISCRETE DYNAMIC MODELS
}

\author{
V.P. MAKSIMOV
}

\begin{abstract}
We consider dynamic models with an aftereffect in the form of functional differential equations with continuous and discrete time. We formulate a general control problem with respect to a given system of target functionals and a brief summary of known results on solvability of this problem under polyhedral point control constraints. In concluding section we present results on estimating the set of attainability under integral restrictions for the control. The proposed version of the synthesis of continuous and discrete systems is based on the systematic use of the theory abstract functional differential equation and has certain advantages in the study of systems and processes with aftereffect. Continuousdiscrete functional-differential models allow us to take into consideration the aftereffects when modeling, including cases of complete memory, and effects arising when impulse perturbations (shocks) are taken into consideration and they are leading to jump changes in the phase state by components with continuous time.
\end{abstract}

Keywords: functional-differential systems, control problems, hybrid systems, set of attainability.

Mathematics Subject Classification: 34K05, 34K30, 34K35, 93B03, 93C23

\section{INTRODUCTION}

Actual applied problems arising in various fields of applications, including problems of analysis, synthesis and control for real technical and social-economic systems, constantly give rise to new types of mathematical models involving ordinary differential equations. These models include dynamic models containing simultaneously phase variables and equations with both continuous and discrete time; such models and corresponding systems are often called hybrid. An interest of researchers in various classes of hybrid models has been steadily increasing over the last 15 years. We just mention here well-known works of Russian and foreign authors, see [3], [5]-[8], [13]-[15], [19], [20], [23], [24], 27]--30], [35]. It should be said that the aim to offer an universal point view on differential equations and equations in finite differences led to creating the theory of dynamical equations on time scales, see, for instance, works [4], [16]-[18], [22].

Despite the presence of plenty of particular results, the general and relatively complete theory of hybrid systems of a sufficiently general form is currently absent. In this paper we discuss a version of the synthesis of continuous and discrete systems based on the theory abstract functional differential equation (AFDE) [21] and having certain advantages in studying systems and processes with an aftereffect. Continuous-discrete functional differential models give an opportunity to cover the aftereffects in real applications, including the cases of complete memory and the effects arising when impulse disturbances are taken into account (shocks) leading to an abrupt change in the phase state with respect to the components with continuous time. One of the main aspects of this problem is the possibility, need and feasibility of using complete results currently known for functional differential systems and systems of difference equations. We note that, with rare exceptions, in papers on hybrid systems, a corresponding continuous time subsystem is described by ordinary differential equations. In contrast to these works,

V.P. Maksimov, Continuous-Discrete Dynamic models.

(C) MaKsimov V.P. 2021.

The reported study was funded by RFBR according to the research project no. 18-01-00332.

Submitted February 15, 2021. 
the models we consider here cover a very general case of differential and integro-differential equations with aftereffect, including systems with impulse actions.

\section{Continuous-Discrete Models With AFterefFeCT}

In order to describe a considered class of models, we first introduce basic spaces. We fix a finite segment $[0, T] \subset \mathbb{R}$. By $L^{n}=L^{n}[0, T]$ we denote the space of summable functions $v:[0, T] \rightarrow \mathbb{R}^{n}$ with the norm

$$
\|v\|_{L^{n}}=\int_{0}^{T}|v(s)|_{n} d s
$$

where $|\cdot|_{n}(|\cdot|$ if the dimension of the space is clear from the context) is the norm in the space $\mathbb{R}^{n}$. By the symbol $L_{2}^{r}=L_{2}^{r}[0, T]$ we denote the space of square summable functions $u:[0, T] \rightarrow \mathbb{R}^{r}$ with the scalar product

$$
(u, v)=\int_{0}^{T} u^{\prime}(s) v(s) d s
$$

where the symbol $(\cdot)^{\prime}$ denotes the transposition.

We define a set $\left\{\tau_{1}, \ldots, \tau_{m}\right\}, 0<\tau_{1}<\ldots<\tau_{m}<T$, and we introduce a space $P A C^{n}(m)=$ $P A C^{n}\left[0, \tau_{1}, \ldots, \tau_{m}, T\right]$, see [1], as the space of piece-wise absolutely continuous functions $x$ : $[0, T] \rightarrow \mathbb{R}^{n}$ which can be represented as

$$
x(t)=\int_{0}^{t} v(s) d s+x(0)+\sum_{k=1}^{m} \chi_{\left[\tau_{k}, T\right]}(t) \Delta x\left(\tau_{k}\right),
$$

where $v \in L^{n}, \Delta x\left(\tau_{k}\right)=x\left(\tau_{k}\right)-x\left(\tau_{k}-0\right), \chi_{\left[\tau_{k}, T\right]}(t)$ is the characteristic function of the segment $\left[\tau_{k}, T\right]:$

$$
\chi_{\left[\tau_{k}, T\right]}(t)=1 \quad \text { as } \quad t \in\left[\tau_{k}, T\right], \quad \chi_{\left[\tau_{k}, T\right]}(t)=0 \quad \text { as } \quad t \notin\left[\tau_{k}, T\right] .
$$

Thus, the elements of the space $P A C^{n}(m)$ are functions absolutely continuous on each of the segments $\left[0, \tau_{1}\right),\left[\tau_{1}, \tau_{2}\right), \ldots,\left[\tau_{m}, T\right]$, right continuous at the points $\tau_{1}, \ldots, \tau_{m}$. Being equipped with the norm

$$
\|x\|_{P A C^{n}(m)}=\|\dot{x}\|_{L^{n}}+|x(0)|_{n}+\sum_{k=1}^{m}\left|\Delta x\left(\tau_{k}\right)\right|_{n}
$$

the space $P A C^{n}(m)$ becomes Banach and isometrically isomorphic to the direct product $L^{n} \times$ $\mathbb{R}^{n+n m}$

The space $A C^{n}=A C^{n}[0, T]$ is the space of absolutely continuous functions $x:[0, T] \rightarrow \mathbb{R}^{n}$ with the norm

$$
\|x\|_{A C^{n}}=\|\dot{x}\|_{L^{n}}+|x(0)|_{n} .
$$

We note that space $P A C^{n}(m)$ is a finite-dimensional extension of the space $A C^{n}$. At that, the jumps $\Delta x\left(\tau_{k}\right)$ of the elements in $P A C^{n}(m)$ can be interpreted as a result of impulse action on the elements of the space $A C^{n}$; a detailed description of such interpretation can be found in 1 .

We fix a set $J=\left\{t_{0}, t_{1}, \ldots, t_{\mu}\right\}, 0=t_{0}<t_{1}<\ldots<t_{\mu}=T$. By $F D^{\nu}(\mu)=$ $F D^{\nu}\left\{t_{0}, t_{1}, \ldots, t_{\mu}\right\}$ we denote the space of functions $z: J \rightarrow \mathbb{R}^{\nu}$ with the norm

$$
\|z\|_{F D^{\nu}}=\sum_{i=0}^{\mu}\left|z\left(t_{i}\right)\right|_{\nu} .
$$

Below we employ the symbol $z$ for the elements of the space $F D^{\nu}$ bearing in mind that $z=$ $\operatorname{col}\left(z\left(t_{0}\right), \ldots, z\left(t_{\mu}\right)\right)$, and we introduce the symbol $\rho z=\operatorname{col}\left(z\left(t_{1}\right), \ldots, z\left(t_{\mu}\right)\right)$. The space of all 
$\rho z, z \in F D^{\nu}$, embedded into $F D^{\nu}(\mu): \rho z \rightarrow \operatorname{col}\left(0, z\left(t_{1}\right), \ldots, z\left(t_{\mu}\right)\right) \in F D^{\nu}$, is denoted by the symbol $F D_{1}^{\nu}$.

While describing continuous-discrete models, we follow work [26]. We write a general case of linear impulse continuous-discrete model as

$$
\begin{aligned}
\dot{x} & =\mathcal{T}_{11} x+\mathcal{T}_{12} z+f, \\
\rho z & =\mathcal{T}_{21} x+\mathcal{T}_{22} z+g,
\end{aligned}
$$

where linear bounded Volterra operators $\mathcal{T}_{i j}, i, j=1,2$, act as follows:

$$
\mathcal{T}_{11}: P A C^{n} \rightarrow L^{n}, \quad \mathcal{T}_{12}: F D^{\nu} \rightarrow L^{n}, \quad \mathcal{T}_{21}: P A C^{n} \rightarrow F D^{\nu}, \quad \mathcal{T}_{22}: F D^{\nu} \rightarrow F D^{\nu} .
$$

We recall that a linear operator $V: X \rightarrow Y$, where $X$ and $Y$ are linear spaces of measurable on $[0, T]$ vector function, is called Volterra operator if for each $\tau \in(0, T)$ and each $x \in X$ such that $x(t)=0$ on $[0, \tau]$, the identity holds $(V x)(t)=0$ on $[0, \tau]$. In the case when the operators $\mathcal{T}_{i j}$ are Volterra ones, system 2.1] is a dynamical model with an afteraffect.

The used representation of these operators is given below. Together with model (2.1), we shall also consider its restriction corresponding to the case when the phase space $P A C^{n}$ of the component $x$ with a continuous time is replaced by the space $A C^{n}$. In this case the impulse action is absent and all $\Delta x\left(\tau_{k}\right)$ vanish. While referring to such model, we shall additional employ the asterisk: $(2.1)^{*}$.

System (2.1) is a particular case of an abstract functional differential equation (AFDE) [21], [1. We recall the definition of AFDE. Let $D$ and $B$ be Banach spaces and $D$ be isomorphic to the direct product $B \times \mathbb{R}^{p}$ (briefly $D=B \times \mathbb{R}^{p}$ ). The equation

$$
\mathcal{L} y=\varphi
$$

with a linear bounded operator $\mathcal{L}: D \rightarrow B$ is called a linear AFDE. The theory of equation (2.2) is systematically exposed in [21], [1]. We fix an isomorphism $J=\{\nabla, \Upsilon\}: B \times \mathbb{R}^{p} \rightarrow D$ and we denote by $J^{-1}=[\delta, r]$ the inverse mapping. Here $\nabla: B \rightarrow D, \Upsilon: \mathbb{R}^{n} \rightarrow D$ and $\delta: D \rightarrow B, r: D \rightarrow \mathbb{R}^{n}$ are corresponding components of the operators $J$ and $J^{-1}$ :

$$
\begin{array}{ll}
J\{v, \alpha\}=\nabla v+\Upsilon \alpha \in D, & v \in B, \quad \alpha \in \mathbb{R}^{p}, \\
J^{-1} y=\{\delta y, r y\} \in B \times \mathbb{R}^{p}, & y \in D .
\end{array}
$$

The system

$$
\delta y=v, \quad r y=\alpha
$$

is called the principal boundary value problem. For each $\{v, \alpha\} \in B \times \mathbb{R}^{p}$, an element

$$
y=\nabla v+\Upsilon \alpha
$$

is a solution of problem (2.3). Representation (2.4) provides that for the operator $\mathcal{L}$ we have

$$
\mathcal{L} y=\mathcal{L}(\nabla v+\Upsilon \alpha)=\mathcal{L} \nabla v+\mathcal{L} \Upsilon Y \alpha=Q v+A \alpha,
$$

the operator $Q: B \rightarrow B$, a so-called principal part of the operator $\mathcal{L}$, and finite-dimensional operator $A: \mathbb{R}^{p} \rightarrow D$ are defined by the identities $Q=\mathcal{L} \nabla$ and $A=\mathcal{L} \Upsilon$. A general theory of equation 2.2 was constructed under the assumption that the operator $Q$ is Fredholm, that is, it can be represented as the sum of an invertible and a completely continuous operators.

From the point of view of the AFDE theory, system (2.1) can be written as equation (2.2) with the operator $\mathcal{L}$ defined by the identity

$$
\mathcal{L} y=\left(\begin{array}{c}
\dot{x}-\mathcal{T}_{11} x-\mathcal{T}_{12} z \\
\rho z-\mathcal{T}_{21} x-\mathcal{T}_{22} z
\end{array}\right)
$$

where $y=\operatorname{col}(x, z) \in P A C^{n} \times F D^{\nu}$. In this case $D=B \times Z, B=L^{n} \times F D_{1}^{\nu}$ and $Z=$ $\mathbb{R}^{n+n m} \times \mathbb{R}^{\nu}$. We also observe that here the operators $\delta$ and $r$ are defined by the identities

$$
\delta y=\operatorname{col}(\dot{x}, \rho z), \quad r y=\operatorname{col}\left(x(0), \Delta x\left(\tau_{1}\right), \ldots, \Delta x\left(\tau_{m}\right), z(0)\right) .
$$


In order to define the principal part $Q: B \rightarrow B$, we introduce the operators

$$
V: L^{n} \rightarrow A C^{n}, \quad U: F D_{1}^{\nu} \rightarrow F D^{\nu}
$$

by the formulae

$$
(V \mathrm{v})(t)=\int_{0}^{t} \mathrm{v}(s) d s, \quad U v=\operatorname{col}(0, v) .
$$

Thus, we arrive at the representation

$$
Q\left(\begin{array}{l}
\mathrm{v} \\
v
\end{array}\right)=\left(\begin{array}{l}
\mathrm{v}-\mathcal{T}_{11} V \mathrm{v}-\mathcal{T}_{12} U v \\
v-\mathcal{T}_{21} V \mathrm{v}-\mathcal{T}_{22} U v
\end{array}\right)
$$

In all cases we consider below, the Fredholm property of the operator $Q$ is implied by its invertibility. The general theory of AFDE answers principal questions on the structure of a general solution of equation (2.1), the dimension of the space of solutions to the homogeneous equation $(f=0, g=0)$, criterions of unique solvability of general boundary value problems. This gives an opportunity to concentrate on a detailed studying actual problems having also an application value, while considering continuous-discrete FDE, employing at the same time the features of this class of dynamical models. In the present work we restrict ourselves by the results obtained while studying control problems.

\section{Control problems}

In order to formulate a general control problem, we introduce control actions into system 2.1):

$$
\begin{aligned}
\dot{x} & =\mathcal{T}_{11} x+\mathcal{T}_{12} z+F u+f, \\
\rho z & =\mathcal{T}_{21} x+\mathcal{T}_{22} z+G u+g,
\end{aligned}
$$

where $F: L_{2}^{r} \rightarrow L^{n}, G: L_{2}^{r} \rightarrow F D^{\nu}$ are linear bounded Volterra operators and we define operators $\mathcal{T}_{i j}, i, j=1,2$, by the identities:

$$
\left(\mathcal{T}_{11} x\right)(t)=\int_{0}^{t} K^{1}(t, s) \dot{x}(s) d s+A_{0}^{1}(t) x(0)+\sum_{k=1}^{m} A_{k}^{1}(t) \Delta x\left(\tau_{k}\right), \quad t \in[0, T],
$$

where the entries $k_{i j}^{1}(t, s)$ of the kernel $K^{1}(t, s)$ are measurable on the set $0 \leqslant s \leqslant t \leqslant T$ and possesses a common majorant $\kappa(t)$ summable on $[0, T]$ :

$$
\left|k_{i j}^{1}(t, s)\right| \leqslant \kappa(t), \quad i, j=1, \ldots, n,
$$

while $(n \times n)$-matrices $A_{0}^{1}, \ldots, A_{m}^{1}$ have summable on $[0, T]$ entries;

$$
\left(\mathcal{T}_{12} z\right)(t)=\sum_{\left\{j: t_{j}<t\right\}} B_{j}^{1}(t) z\left(t_{j}\right), \quad t \in[0, T]
$$

where the entries of the matrices $B_{j}^{1}, j=0, \ldots, \mu$, are summable on $[0, T]$; as usually, hereinafter $\sum_{i=k}^{l} P_{i}=0$ for all $P_{i}$ if $l<k$ :

$$
\left(\mathcal{T}_{21} x\right)\left(t_{i}\right)=\int_{0}^{t_{i-1}} K_{i}^{2}(s) \dot{x}(s) d s+A_{i 0}^{2} x(0)+\sum_{k: \tau_{k}<t_{i}} A_{i k}^{2} \Delta x\left(\tau_{k}\right), \quad i=1, \ldots, \mu,
$$


where the entries of the matrices $K_{i}^{2}$ are measurable and essentiable bounded on $[0, T],(\nu \times n)$ matrices $A_{i k}^{2}, i=1, \ldots, \mu, k=1, \ldots, m$, are constant;

$$
\left(\mathcal{T}_{22} z\right)\left(t_{i}\right)=\sum_{j=0}^{i-1} B_{i j}^{2} z\left(t_{j}\right), \quad i=1, \ldots, \mu,
$$

where $B_{i j}^{2}$ are constant $(\nu \times \nu)$-matrices.

The initial state of the control system is supposed to be given:

$$
x(0)=\alpha, \quad z(0)=\delta .
$$

The target of the control is defined by the identity

$$
\ell y=\ell\left(\begin{array}{l}
x \\
z
\end{array}\right)=\beta, \quad \beta \in \mathbb{R}^{N},
$$

where $\ell: P A C^{n}(m) \times F D^{\nu}(\mu) \rightarrow \mathbb{R}^{N}$ is a linear bounded vector functional. We recall a representation for such vector functional:

$$
\ell\left(\begin{array}{l}
x \\
z
\end{array}\right)=\int_{0}^{T} \Phi(s) \dot{x}(s) d s+\Psi_{0} x(0)+\sum_{k=1}^{m} \Psi_{k} \Delta x\left(\tau_{k}\right)+\sum_{j=0}^{\mu} \Gamma_{j} z\left(t_{j}\right) .
$$

Here $\Psi_{k}, k=0,1, \ldots, m$, are constant $(N \times n)$-matrices, $\Gamma_{j}, j=0,1, \ldots, \mu$, are constant $(N \times \nu)$-matrices, $\Phi$ is a $(N \times n)$-matrix with measurable and essentially bounded on $[0, T]$ entries. We assume that the entries $\ell_{i}: P A C^{n}(m) \times F D^{\nu}(\mu) \rightarrow \mathbb{R}, i=1, \ldots, N$, are linearly independent.

In the case, when the control $u \in L_{2}^{r}$ and an impulse control, the role of which is played by the jumps $\Delta x\left(\tau_{k}\right), k=1, \ldots, m$, of the trajectory $x \in P A C^{n}(m)$, obey no constraints, the solvability criterion of problem $(3.1)-(3.3)$ in the class of program control was formulated in [26].

In the case of polyhedral point restrictions for the control $u$ :

$$
\Lambda \cdot u(t) \leqslant \gamma, \quad \gamma \in \mathbb{R}^{N_{1}}, \quad t \in[0, T]
$$

in a natural way, there arises a problem on describing the set of all target values $\beta$, for which control problem (3.1)-(3.4) is solvable. The mentioned set is called an attainability set for this problem. We note that in the most part of works on attainability sets for controlled dynamical models the attainability is understood for the terminal values of the trajectory, while in our work it is understood for a target vector functional $\ell$ of a general form ( $\ell$-attainability). The main constructions for obtaining estimates for the sets of $\ell$-attainability are based on using the results from [31] on studying a general moment problem and they are presented in work 32] for the case, when the impulse part of the control is absent. The case with the impulse component was considered in 9. All constructions used for obtaining internal (lower by inclusion) and external (upper by inclusion) estimates for the attainability set were essentially based on a representation for the solution for the Cauchy problem of system (2.1):

$$
y=\mathcal{Y}\left(\begin{array}{l}
\alpha \\
\delta \\
\sigma
\end{array}\right)+\mathcal{C}\left(\begin{array}{l}
f \\
g
\end{array}\right)
$$

Here $\mathcal{Y}$ is a fundamental matrix of solutions of a homogeneous system, $\mathcal{C}=\left(\begin{array}{ll}\mathcal{C}_{11} & \mathcal{C}_{12} \\ \mathcal{C}_{21} & \mathcal{C}_{22}\end{array}\right)$ is the Cauchy operator, $\sigma=\operatorname{col}\left(\Delta x\left(\tau_{1}\right), \ldots, \Delta x\left(\tau_{m}\right)\right)$. The structure of the Cauchy operator, the representations and the properties of its components were studied [33], [10], while in works [11], [34] there was selected and studied a class of continuous-discrete models with a discrete memory, for which the Cauchy operator is constructed explicitly by the parameters of the model. In this 
class, it is possible to solve explicitly the problem on constructing program controls leading to given attainable target values [12]. Applications of continuous-discrete models to problems of economy dynamics were discussed in [11], [2], [25].

\section{ESTIMATE OF ATTAINABILITY SET FOR CONTROL PROBLEM WITH INTEGRAL CONSTRAINTS}

We consider the control problem $(3.1)^{*}-3.3$ with integral constraints on a control $u \in L_{2}^{r}$ :

$$
\int_{0}^{T} \Lambda(t) \cdot u(t) d t \leqslant \gamma, \quad \gamma \in \mathbb{R}^{N_{1}}
$$

where $\Lambda(\cdot)$ is a $\left(N_{1} \times r\right)$-matrix, the entries of which are square summable on $[0, T]$. We recall that the asterisk ${ }^{*}$ in the reference $(3.1)^{*}$ indicates the restriction to the space $A C^{n}$ of all operators acting on the component $x$ of the phase vector $y=\operatorname{col}(x, z)$ and in this way the impulse control component is excluded.

We employ representation (3.5) and obtain an expression for the values of the target vector functional on all trajectories generated by controls $u \in L_{2}^{r}$ :

$$
\begin{aligned}
\ell\left(\begin{array}{l}
x \\
z
\end{array}\right)= & \int_{0}^{T} \Phi(s) \frac{d}{d s}\left(\mathcal{C}_{11} f+\mathcal{C}_{12} g+\mathcal{C}_{11} F u+\mathcal{C}_{12} G u\right) d s+\Psi_{0} \alpha+\Gamma_{0} \delta \\
& +\Gamma\left(\mathcal{C}_{21} f+\mathcal{C}_{22} g+\mathcal{C}_{21} F u+\mathcal{C}_{22} G u\right),
\end{aligned}
$$

where $\Gamma=\left(\Gamma_{1}, \ldots, \Gamma_{\mu}\right)$.

We collect terms involving the control:

$$
\ell\left(\begin{array}{l}
x \\
z
\end{array}\right)=\int_{0}^{T} \Phi(s) \frac{d}{d s}\left(\mathcal{C}_{11} F u+\mathcal{C}_{12} G u\right) d s+\Gamma\left(\mathcal{C}_{21} F u+\mathcal{C}_{22} G u\right)+\widetilde{\beta},
$$

where

$$
\widetilde{\beta}=\int_{0}^{T} \Phi(s) \frac{d}{d s}\left(\mathcal{C}_{11} f+\mathcal{C}_{12} g\right) d s+\Gamma\left(\mathcal{C}_{21} f+\mathcal{C}_{22} g\right)+\Psi_{0} \alpha+\Gamma_{0} \delta
$$

is the sum of terms containing no control variable and reflecting the dependence on the initial state of the system and on given external actions.

For further calculations we employ a representation of the components of the Cauchy operator constructed in [33]:

$$
\begin{aligned}
& \left(\mathcal{C}_{11} f\right)(t)=\int_{0}^{t} C_{11}(t, s) d s, \quad\left(\mathcal{C}_{12} g\right)(t)=\sum_{l: t_{l} \leqslant t} \int_{0}^{t} C_{12}(t, s) d s g\left(t_{l}\right), \quad t \in[0, T] ; \\
& \left(\mathcal{C}_{21} f\right)\left(t_{i}\right)=\int_{0}^{t_{i}} C_{21}(i, s) f(s) d s, \quad\left(\mathcal{C}_{22} g\right)\left(t_{i}\right)=\sum_{l=1}^{i} C_{i, l} g\left(t_{l}\right), \quad i=1, \ldots, \mu .
\end{aligned}
$$

The expressions for the kernels $C_{i j}, i, j=1,2$, can be found in the mentioned work. Employing these representations, identity (3.3) defining the target of the control can be reduced to the integral form with respect to the control $u$ :

$$
\int_{0}^{T} M(t) \cdot u(t) d t=\beta-\widetilde{\beta}
$$


where $M(t)$ is an $(N \times r)$-matrix (moment matrix) and the constant vector $\widetilde{\beta}$ is defined by identity (4.2). Thus, control problem with integral restrictions for the control is reduced to the system

$$
\int_{0}^{T} M(t) \cdot u(t) d t=(\beta-\widetilde{\beta}) \in \mathbb{R}^{N}, \quad \int_{0}^{T} \Lambda(t) \cdot u(t) d t \leqslant \gamma \in \mathbb{R}^{N_{1}} .
$$

A lower by inclusion estimate for the attainability set is based on the following constructions. We partition the segment $[0, T]$ into partial intervals by the points $\vartheta_{1}, \ldots, \vartheta_{\mathcal{K}-1}$ :

$$
0=\vartheta_{0}<\vartheta_{1}<\ldots<\vartheta_{\mathcal{K}-1}<T=\vartheta_{\mathcal{K}}
$$

and we denote by $\chi_{i}(t)$ the characteristic function of the interval $\left(\vartheta_{i-1}, \vartheta_{i}\right]$. We restrict the class of used controls by piecewise constant controls of the form

$$
u(t)=\sum_{i=1}^{\mathcal{K}} d_{i} \chi_{i}(t)
$$

where $d_{i} \in \mathbb{R}^{r}$ are constant vectors. We define constant $(N \times r)$-matrices $M_{i}$ and $\left(N_{1} \times r\right)$ matrices $\Lambda_{i}$ by the identities

$$
M_{i}=\int_{\vartheta_{i-1}}^{\vartheta_{i}} M(t) d t, \quad \Lambda_{i}=\int_{\vartheta_{i-1}}^{\vartheta_{i}} \Lambda(t) d t .
$$

We fix a set of vectors $\lambda_{1}, \ldots, \lambda_{j}, \ldots, \lambda_{\mathcal{N}} \in \mathbb{R}^{N}$ and for each $j$ we formulate the problem of the linear programming:

$$
\sum_{i=1}^{\mathcal{K}} \lambda_{j}^{\prime} \cdot M_{i} d_{i} \rightarrow \max , \quad \sum_{i=1}^{\mathcal{K}} \Lambda_{i} d_{i} \leqslant \gamma .
$$

Let $\lambda_{j_{1}}, \ldots, \lambda_{j_{\mathcal{N}_{1}}}$ be a subset of the set $\left\{\lambda_{j}\right\}, j=1, \ldots, \mathcal{N}$, and for each its entry problem (4.6) has a solution $\mathcal{D}^{j_{k}}=\left(d_{1}^{j_{k}}, \ldots, d_{\mathcal{K}}^{j_{k}}\right), k=j_{1}, \ldots, j_{\mathcal{N}_{1}}$. While substituting each such solution into (4.5), we define a program control $u^{j_{k}}(t)$, which gives an attainable value of the target vector functional $\ell$ :

$$
\ell\left(\begin{array}{l}
x \\
z
\end{array}\right)=\int_{0}^{T} M(t) \cdot u^{j_{k}}(t) d t=\rho^{j_{k}} .
$$

The set of these values, being the points in the space $\mathbb{R}^{N}$, allows us to provide an internal estimate for the attainability set.

Theorem 4.1. Let $P$ be the set of all convex combinations of the points $\rho^{j_{k}}, k=j_{1}, \ldots, j_{\mathcal{N}_{1}}$. Then each value $\beta \in \mathbb{R}^{N}$ such that the point $\rho=\beta-\widetilde{\beta}$ with the constant vector $\widetilde{\beta}$ defined by identity (4.2) belongs to the set $P$ is an attainable value of the target functional $\ell$ in problem $3.1{ }^{*}-3.3$, 4.1 .

Proof. Each control $u^{j_{k}}(t)$ satisfies integral constraint 4.1). Each control

$$
u(t)=\sum_{k=1}^{\mathcal{N}_{1}} \omega_{k} u^{j_{k}}(t)
$$

satisfies this constraint at well; here

$$
\omega_{k} \geqslant 0, \quad \sum_{k=1}^{\mathcal{N}_{1}} \omega_{k}=1 .
$$


Indeed, it follows from

$$
\int_{0}^{T} \Lambda(t) u^{j_{k}}(t) d t \leqslant \gamma
$$

that

$$
\omega_{k} \int_{0}^{T} \Lambda(t) u^{j_{k}}(t) d t=\int_{0}^{T} \Lambda(t) \omega_{k} u^{j_{k}}(t) d t \leqslant \omega_{k} \gamma, \quad k=1, \ldots, \mathcal{N}_{1} .
$$

Summing up these inequalities, we obtain

$$
\int_{0}^{T} \Lambda(t) u(t) d t \leqslant \gamma
$$

In order to complete the proof, it remains to observe that each point in the set $P$ determined by a fixed set of the coefficients of the convex combination corresponds to a program control with the same set of the coefficients at the controls $u^{j_{k}}(t)$.

\section{BIBLIOGRAPHY}

1. N.V. Azbelev, V.P. Maksimov, L.F. Rakhmatullina. Introduction to the theory of functional differential equations. Methods and applications. Inst. Comput. Studies, Moscow (2002). [Hindawi Publishing Corp, New York (2007).]

2. D.L. Andrianov, V.O. Arbuzov, S.V. Ivliev, V.P. Maksimov, P.M. Simonov. Ecomomic dynamics models: theory, applications, computer aided implementation // Vestnik Permskogo Univ. Economika. 4(27), 8-32 (2015). [Perm University Herald. Economy. 4(27), 33-53 (2015).]

3. V.A. Baturin, E.V. Goncharova, N.S. Maltugueva. Iterative methods for solution of problems of optimal control of logic-dynamic systems // Izv. RAN. Teor. sist. upravl. 5, 53-61 (2010). [J. Comp. Syst. Sci. Int. 49:5, 731-739 (2010).]

4. Zh. I. Bakhtina. Some questions of the theory of dynamic models in time scales // Aktual. Napr. Nauch. Issl. XXI veka: Teor. Prakt. 2:5-1, 19-22 (2014). (in Russian).

5. A.S. Bortakovskii. Necessary optimality conditions for control of logical-dynamical systems // Izv. RAN. Teor. sist. upravl. 6, 16-33 (2007). [J. Comp. Syst. Sci. Int. 46:6, 854-871 (2007).]

6. A.S. Bortakovskii. Optimal and suboptimal control for sets of trajectories of deterministic continuous-discrete systems // Izv. RAN. Teor. sist. upravl. 1, 18-33 (2009). [J. Comp. Syst. Sci. Int. 48:1, 14-29 (2009).]

7. A.S. Bortakovskii. Optimal and suboptimal control of bundle of trajectories of deterministic logical-dynamical systems // Izv. RAN. Teor. sist. upravl. 6, 29-46 (2009). [J. Comp. Syst. Sci. Int. 48:6, 873-890 (2009).]

8. R. Gabasov, N.S. Paulianok, F.M. Kirillova. Optimal control of some hybrid systems // Izv. RAN. Teor. sist. upravl. 6, 2-52 (2010). [J. Comp. Syst. Sci. Int. 49:6, 872-882 (2010).]

9. V.P. Maksimov. Attainable values of on-target functionals for a functional differential system with impulse action // Vestn. Tambovskogo Univ. Ser. Estestv. Tekn. Nauki. 23:123, 441-447 (2018). (in Russian).

10. V.P. Maksimov. On the construction and estimates of the Cauchy matrix for systems with aftereffect // Trudy Inst. Matem. Mekh. UrO RAN. 25:3, 153-162 (2019). (in Russian).

11. V.P. Maksimov. Attainable values of on-target functionals in economic dynamics problems // Prikl. Matem. Vopr. Upravl. 4, 124-135 (2019). (in Russian).

12. V.P. Maksimov. On the construction of program control in the problem on attainable values of on-target functionals for dynamic economic models with discrete memory // Prikl. Matem. Vopr. Upravl. 3, 89-104 (2020). (in Russian).

13. V.M. Marchenko, Z. Zaczkiewicz. Solution representations for controlled hybrid differentialdifference impulsive systems // Diff. Uravn. 45:12, 1775-1786 (2009). [Diff. Equat. 45:12, 17761786 (2009).] 
14. V.M. Marchenko, O.N. Poddubnaya. Solution representations and relative controllability of linear differential algebraic systems with several delays // Dokl. RAN. 404:4, 465-469 (2005). [Doklady Math. 72:2, 824-828 (2005).]

15. S.A. Minyuk, O.A. Panasik. Controllability and attainability criteria for linear differential- algebraic systems // Izv. RAN. Teor. sist. upravl. 5, 5-18 (2008). [J. Comp. Syst. Sci. Int. 47:5, 673-686 (2008).]

16. Yu.V. Pokornyi, Zh.I. Bakhtina. On the Stieltjes procedure for closing gaps in time scales // Matem. Zamet. 86:5, 733-735 (2009). [Math. Notes. 86:5, 690-692 (2009).]

17. R. Agarwal, M. Bohner, D. O'Regan, A. Peterson. Dynamic equations on time scales: A survey // J. Comp. Appl. Math. 141:1-2, 1-26 (2002).

18. R.P. Agarwal, M. Bohner, A. Boichuk, O. Strakh. Fredholm boundary value problems for perturbed systems of dynamic equations on time scales // Math. Meth. Appl. Sci. 38:17, 4178-4186 (2015).

19. G. Agranovich. Observability criteria of linear discrete-continuous LTI systems with continuoustime measurements // Funct. Diff. Equats. 16:1, 35-51 (2009).

20. G. Agranovich. Observer for discrete-continuous LTI systems with continuous-time measurements // Funct. Diff. Equats. 18:1-2, 3-12 (2011).

21. N.V. Azbelev, L.F. Rakhmatullina. Theory of linear abstract functional-differential equations and applications // Memoirs Diff. Equat. Math. Phys. 8, 1-102 (1996).

22. M. Bohner, A. Peterson. Advances in dynamic equations on time scales, Birkhäuser, Boston (2003).

23. A.S. Bortakovski. Optimal and suboptimal control for sets of trajectories of deterministic continuous discrete systems // J. Comp. Syst. Sci. Int. 48:1, 14-29 (2009).

24. M. Branicky, V. Borkar, S. Mitter. A unified framework for hybrid control: Model and optimal control theory // IEEE Trans. Autom. Contr. 43, 31-45 (1998).

25. E.I. Bravyi, V.P. Maksimov, P.M. Simonov. Some economic dynamics problems for hybrid models with aftereffect // Math. 8:10, 1832 (2020).

26. A.L. Chadov, V.P. Maksimov. Linear boundary value problems and control problems for a class of functional differential equations with continuous and discrete times // Funct. Diff. Equatns. 19:1-2, 49-62 (2012).

27. F. Clarke, R. Vinter. Optimal multiprocesses// SIAM J. Control Optim. 27:5, 1072-1091 (1989).

28. M. De la Sen. On the controller synthesis for linear hybrid systems // IMA J. Math. Contr. Inform. 18:4, 503-529 (2001).

29. M. De la Sen. Identification of a class of hybrid systems // IMA J. Math. Contr. Inform. 20:3, $233-261(2003)$.

30. A. Ichikava, H. Katayama. Linear time varying systems and sampled-data systems. SpringerVerlag, London (2001).

31. M.G. Krein, A.A. Nudel'man. The Markov moment problem and extremal problems. Amer. Math. Soc., New York (1977).

32. V.P. Maksimov. On the $\ell$-attainability sets of continuous discrete functional differential systems // IFAC-PapersOnLine. 51:32, 310-313 (2018).

33. V.P. Maksimov. The structure of the Cauchy operator to a linear continuous-discrete functional differential system with aftereffect and some properties of its components // Vestnik Udmurtskogo Universiteta. Matematika. Mekhanika. Komp'yuternye Nauki. 29:1, 40-51 (2019). (in Russian).

34. V.P. Maksimov. On a class of linear continuous-discrete systems with discrete memory // Vestnik Udmurtskogo Universiteta. Matematika. Mekhanika. Komp'yuternye Nauki. 30:3, 385-395 (2020). (in Russian).

35. D. Sworder, J. Boid. Estimation problems in hybrid systems. Cambridge University Press, Cambridge (2000).

Vladimir Petrovich Maksimov,

Perm State National University,

Bukireva str. 15,

614990, Perm, Russia

E-mail: maksimov@econ.psu.ru 